\title{
CARACTERIZACIÓN ENERGÉTICA Y DETERMINACIÓN DEL POTENCIAL DE GENERACIÓN ELÉCTRICA CON TECNOLOGÍAS TERMOQUÍMICAS A PARTIR de residuos degradables en Santiago de los Caballeros (República Dominicana)
}

\author{
Energy Characterization and Determination of the Electricity \\ Generation Potential with Thermochemical Technologies Using the \\ Degradable Household Waste Fraction in Santiago de los Caballeros \\ (Dominican Republic)
}

\author{
Julian Cuervo a Margarita Betances $^{\mathrm{b}}$ y Esclaudys Perez ${ }^{\mathrm{c}}$
}

Recibido: 7/7/2021 Aprobado: 1/10/2021

Cómo citar: Cuervo, J., Betances, M., \& Peréz, E. (2021). Caracterización energética y determinación del potencial de generación eléctrica con tecnologías termoquímicas a partir de residuos degradables en Santiago de los Caballeros (República Dominicana). Ciencia, Ingenierías y Aplicaciones, 4(2), 21-39. Doi: https://doi. org/10.22206/cyap.2021.v4i2.pp21-39

\section{Resumen}

Este artículo presenta resultados donde se caracteriza energéticamente la fracción degradable de los residuos domésticos mediante la prueba de poder calorifico, además se determinó el potencial de generación eléctrica de esta fracción con tecnologías termoquímicas en un caso de estudio para la ciudad de Santiago de los Caballeros, República Dominicana. De acuerdo con los resultados, el poder calorifico promedio arrojó un valor de $9.337,6 \mathrm{~kJ} \cdot \mathrm{kg}-1$. El valor más alto fue obtenido por los residuos de jardinería $10.560,6 \mathrm{~kJ} \cdot \mathrm{kg}-1$. Seguido por los residuos de alimentos $10.385,4 \mathrm{~kJ} \cdot \mathrm{kg}-1$. Los desechos de madera, cartón y papel arrojaron $9.875,0 \mathrm{~kJ} \cdot \mathrm{kg}-1,9.227,4 \mathrm{~kJ} \cdot \mathrm{kg}-1$ y $6.639,7 \mathrm{~kJ} \cdot \mathrm{kg}-1$, respectivamente. El potencial de generación bruta de electricidad por día con respecto a los desechos orgánicos alcanzó un máximo de 308,8 MWh por día a través de la Gasificación de Ciclo Combinado (GCC).

Palabras clave: residuos degradables; valor calorífico; potencial eléctrico; residuos domésticos.

\footnotetext{
a Encargado Laboratorio de Energías Renovables, facultad de Ciencias Agroalimentarias y Medio Ambiente, Universidad ISA, Santiago de los Caballeros, República Dominicana.

ORCID: 0000-0002-2827-1887, Correo-e: julianm.cuervo@gmail.com

b Profesora Investigadora, departamento de Recursos Naturales, Universidad ISA.

Correo-e: mbetances@isa.edu.do

c Profesor Investigador, Departamento de Recursos Naturales, Universidad ISA.

ORCID: 0000-0003-0465-6261, Correo-e: eperez@isa.edu.do
} 


\begin{abstract}
This article presents results where the degradable fraction of household waste is energetically characterized by the calorific value test, in addition the electricity generation potential of this fraction was determined with thermochemical technologies in a case study for the city of Santiago de los Caballeros, Dominican Republic. According to the results, the average calorific value yielded a value of $9,337.6 \mathrm{~kJ} \cdot \mathrm{kg}-1$. The highest value was obtained for garden waste $10,560.6 \mathrm{~kJ} \cdot \mathrm{kg}-1$. Followed by food residues $10,385.4 \mathrm{~kJ} \cdot \mathrm{kg}-1$. Wood 9,875.0 kJ.kg-1, cardboard, 9,227.4 kJ.kg-1, and paper $6,639.7 \mathrm{~kJ} \cdot \mathrm{kg}-1$, respectively. The gross generation potential of electricity per day with respect to organic waste reached a maximum of $308.8 \mathrm{MWh}$ per day through Combined Cycle Gasification (CCG).
\end{abstract}

Keywords: Household waste; degradable waste; calorific value; electrical potential. 


\section{Introducción}

Existe una creciente preocupación ambiental con respecto al impacto contaminante e insostenibilidad de los combustibles fósiles. El uso de materia orgánica para la energía es una herramienta fundamental para un futuro sostenible. Además, depositar estos materiales en vertederos como práctica habitual en República Dominicana representa un gran desperdicio de energía utilizable. Otro factor clave es que a nivel mundial la principal fuente de generación de energía son los combustibles fósiles, que rondan el $80 \%$ (Banco Mundial, 2019).

República Dominicana depende en gran medida de los combustibles fósiles para la generación de energía. El país importa el $85 \%$ de su combustible, a pesar de tener abundantes residuos y biomasa que podrían representar una sustitución importante para estas importaciones (Comisión Nacional de Energía -CNE, 2017). Según The Economist Intelligence Unit (2015), uno de los mayores desafíos del sector eléctrico dominicano es diversificar la matriz de generación de energía, reduciendo la participación de combustibles derivados del petróleo y aumentando la participación de fuentes alternativas como las energías renovables o el gas natural. La implementación de tales tecnologías requiere datos científicos confiables, al igual que conocimientos técnicos para funcionar con éxito (Steffen \& Szolar, 1998; Gautam, 2014)

Debido a restricciones técnicas, políticas yeconómicas, sehadesarrollado una amenaza ambiental sin precedentes en República Dominicana, que requiere una intervención inmediata. Esta amenaza se identifica como un riesgo socio-ecológico causado por la falta de disposición y tratamiento efectivo de los desechos sólidos. Se informa que miles de toneladas de desechos orgánicos en descomposición, combinados con otros tipos de desechos, contaminan el aire, el agua y el suelo. Además, existe una presión internacional creciente para la reducción de las emisiones de gases de efecto invernadero donde los rellenos sanitarios representan una adición sustancial (United Nations Climate Change, 2016).

El bajo uso de biomasa residual y desechos biodegradables para generar energía en República Dominicana es preocupante, ya que muchos 
otros países han reconocido el potencial y ya están adoptando tecnologías alternativas para generar bioenergía (Worldwatch Institute, 2015). Según la Organización de las Naciones Unidas para la Alimentación y la Agricultura (2018), la biomasa es considerada una de las fuentes de energía más confiables, por ser constante y almacenable, lo que facilita la generación de energía térmica y eléctrica. Para la implementación de estas tecnologías se requiere recopilar información sobre las características del residuo, la cantidad disponible, el poder calorífico y la ubicación. Esta información es fundamental para contribuir en la ejecución de proyectos relacionados con bioenergía, implementación e investigación de residuos para su aprovechamiento energético. Lamentablemente, el acceso a esta información es limitado o inexistente en el país (Dutta et al., 2014). Una gran desventaja del uso de residuos para la generación de energía es el alto costo de la inversión inicial debido a la gran variabilidad de estos.

Por otro lado, cuando se obtiene un suministro homogéneo de residuos, se pueden obtener resultados exitosos. Un ejemplo es el proyecto ejecutado por el Consorcio Azucarero de San Pedro de Macorís y la empresa San Pedro Bioenergía. Este proyecto cuenta con un sistema de cogeneración que utiliza bagazo de caña de azúcar, inyectando $30 \mathrm{MW}$ a la red nacional y utilizando 12MW para las necesidades energéticas del ingenio (Comisión Nacional de Energía -CNE, 2017). Este estudio tiene como propósito realizar un diagnóstico del potencial energético de los residuos sólidos urbanos biodegradables en Santiago de los Caballeros, utilizando la prueba de poder calorífico y la estimación del potencial eléctrico y centrándose en tecnologías de conversión termoquímica de residuos a energía. El estudio busca dar una respuesta considerando la situación de urgencia provocada por el aumento en la generación de residuos sólidos orgánicos depositados en botaderos abiertos sin ningún tipo de tratamiento (Ministerio de Medio Ambiente y Recursos Naturales, 2014).

Esta investigación pretende coadyuvar a la generación de información sobre residuos urbanos que contribuya a la utilización de los mismos para una mayor oferta de bioenergía, reducir la dependencia de las importaciones de combustibles fósiles, agregar valor a los subproductos municipales y agroindustriales, reducir el alto impacto del metano $\left(\mathrm{CH}_{4}\right)$ $y$ del gas hilarante $\left(\mathrm{N}_{2} \mathrm{O}\right)$ en el calentamiento global y, de esta manera, 
generar conocimiento técnico y una transición de estas tecnologías en las ciudades y comunidades que mejoraría varios aspectos sociales, económicos y ecológicos (CNE, 2009).

\section{Materiales y métodos}

\section{Diagnóstico de producción y composición de residuos domésticos}

Para analizar el potencial energético de la biomasa en Santiago de los Caballeros fue necesario identificar la cantidad de residuos orgánicos producidos. Para identificar la cantidad de residuos se realizó una evaluación para estimar la cantidad y tipo de residuos producidos en Santiago de los Caballeros. Utilizando la fórmula estadística definida para la selección de muestras en poblaciones finitas dadas por McClave y Dietrich (1979), las muestras se determinaron en tres sectores económicos de la clase alto ingreso, clase medio ingreso y clase bajo ingreso:

$$
n=\frac{Z_{1-\alpha / 2}^{2} N \sigma^{2}}{(N-1) E^{2}+Z_{1-\alpha / 2}^{2} N \sigma^{2}}
$$

donde:

$m=$ Tamaño de la muestra (Número de hogares a muestrear)

$N=$ Población del sector (Número de hogares totales en el sector)

$\sigma=$ Desviación Estándar $=0.20$

$E=$ Margen de Error $=0.079$

$Z=$ Valor de distribución Normal $=1.564$ para $90 \%$ probabilidad

Los residuos se clasificaron de la siguiente manera: fracción biodegradable (residuos alimentarios, residuos de jardinería, madera, cartón y papel) y una fracción no degradable (vidrio, tetrapack, metales, aluminio, espuma, textiles, otros, botellas de PET, otros plásticos, residuos peligrosos). Solo los residuos biodegradables se consideraron para la cuantificación de energía. 


\section{Análisis y poder calorífico de la fracción degradable de residuos domésticos}

Se llevaron a cabo cuatro repeticiones para cada prueba. Siguiendo el protocolo de prueba estándar ASTM, se determinaron análisis inmediatos para la humedad ASTM E871, volátiles ASTM E872, cenizas ASTM D1102. El carbono fijo se calculó restando la fracción de volátiles y cenizas del peso inicial de la muestra. El valor calorífico superior se determinó mediante el procedimiento D5865 del método de prueba estándar ASTM para un valor calorífico superior con una bomba calorimétrica de oxígeno normal Parr 1341. Cada muestra se secó en un horno a 100 ${ }^{\circ} \mathrm{C}$ hasta que no hubo pérdida de peso debido a la evaporación. A partir de los datos obtenidos, se calculó el menor valor calorífico, utilizando el llamado método práctico en el que restando el calor de la vaporización de la humedad presente en el residuo al mayor valor calorífico. Para los cálculos de los residuos de alta humedad, se asumió una humedad del 30 \% como la tolerancia máxima de trabajo. No se consideró la fracción de hidrógeno presente en los residuos secos. Se utilizó la siguiente ecuación para los cálculos (Fernández, 2019):

$$
\text { PCI }\left(\frac{\text { Kcal }}{k g}\right)=\operatorname{PCS}\left(\frac{\text { Kcal }}{k g}\right)-597\left(\frac{\text { Pagua }}{\text { Pcomb }}\right)
$$

donde:

PC1 = Poder Calorífico Inferior

PCS= Poder Calorífico Superior

Pagua $=$ Peso en kg del agua presente en la muestra

Pcomb= Peso en kg de la muestra

Kcal=Kilo calorías

$\mathrm{Kg}=$ Kilogramos 
Cálculo del potencial energético de la fracción biodegradable de residuos domésticos

El potencial energético de la fracción degradable de los residuos se determinó utilizando las tecnologías de transformación termoquímica más reconocidas. Se utilizaron los factores que se presentan a continuación en la tabla 1.

\section{Tabla 1}

Eficiencia eléctrica equivalente para procesos de conversión termoquímica

Proceso

\begin{tabular}{ll}
\hline Combustión directa (DC) & 19,9 \\
\hline Gasificación con turbina de gas (GGT) & $25-28$ \\
\hline Gasificación con ciclo combinado (CCG) & $35-40$ \\
\hline Pirólisis con ciclo combinado (PCC) & 31
\end{tabular}

Nota. Serrano, 2017

Para el Valor Bruto de Calor disponible en Santiago de los Caballeros y el potencial eléctrico se dan por las siguientes ecuaciones (Breton et al., 2012; Serrano, 2017):

$$
\mathrm{Q}=\mathrm{m} \cdot \mathrm{PCI}(3)
$$

donde $\mathrm{m}$ es el peso de los residuos disponible y PCI es el Poder Calorífico Inferior

$$
\mathrm{P}=\mathrm{K} \cdot \mathrm{Q} \cdot \eta(4)
$$

donde $\mathrm{K}$ es una constante de conversión de $\mathrm{kJ} \cdot \mathrm{kg}^{-1}$ a KW.h y $\eta$ es la eficiencia de salida eléctrica que se da en la tabla tabla 1. 


\section{Resultados}

La producción y caracterización de residuos sólidos domiciliarios por persona y la composición de estos residuos en porcentaje se muestran en la tabla 2. Analizando siete días en los que se tomaron datos en una muestra compuesta de 20 hogares para cada sector económico, para un total de 60 hogares y 289 personas, se obtuvo una producción media de residuos por habitante y día de $0,799 \mathrm{~kg}$. La fracción degradable equivale al $71.1 \%$ y la fracción no degradable al $28.9 \%$. Entre los residuos de interés para el estudio energético que se muestran en la tabla 2 , los residuos alimentarios constituyen el $49.1 \%$ del compuesto, principalmente arroz y tubérculos y cáscaras de banano. La jardinería representa un 13,9\% compuesta por pasto y hojas de diferentes especies arbóreas como Leucaena leucocephala, Delonix regia y diferentes especies decorativas. La madera, con un $2.0 \%$, provino de las ramas de las especies arbóreas mencionadas y muebles de madera. El papel, con un $3.5 \%$, compuesto principalmente por hojas blancas y periódicos. Cartón, con un 2,8 \%, compuesto por cajas de productos alimenticios.

\section{Tabla 2}

Composición física de residuos domésticos en Santiago de los Caballeros, 2019

\section{Tipo de residuo}

Kg + D.S*

$\%$

Fracción degradable

\begin{tabular}{lrrr}
\hline Desperdicio de alimentos & 0,392 & \pm 0.235 & 49,062 \\
\hline Residuos de jardinería & 0,111 & \pm 0.230 & 13,878 \\
\hline Madera & 0,016 & \pm 0.109 & 1,969 \\
\hline Papel & 0,028 & \pm 0.076 & 3,451 \\
\hline Cartón & 0,022 & \pm 0.038 & 2,772 \\
\hline
\end{tabular}

Fracción no degradable

\begin{tabular}{lccc}
\hline Vidrio & 0,036 & \pm 0.057 & 4,459 \\
\hline Tetrapack & 0,012 & \pm 0.028 & 1,516 \\
\hline Metales & 0,010 & \pm 0.014 & 1,260 \\
\hline Aluminio & 0,001 & \pm 0.002 & 0,079 \\
\hline Espuma (EPS) & 0,015 & \pm 0.041 & 1,933 \\
\hline Textil & 0,006 & \pm 0.012 & 0,754 \\
\hline Otros & 0,022 & \pm 0.048 & 2,767 \\
\hline
\end{tabular}




\begin{tabular}{lrrr}
\hline Tipo de residuo & Kg + D.S* & \multicolumn{1}{c}{$\%$} \\
\hline Botellas de plástico & 0,039 & \pm 0.049 & 4,872 \\
\hline Residuos plásticos & 0,047 & \pm 0.090 & 5,824 \\
\hline Residuos peligrosos & 0,043 & \pm 0.055 & 5,404 \\
\hline Total & 0,799 & \pm 0.563 & 100,000 \\
\hline
\end{tabular}

*Desviación Estandar

\section{Análisis de los resultados}

Los resultados presentados en la tabla 3 determinaron diferentes factores al seleccionar un tipo de tecnología y equipo. Contenido de humedad: los residuos de alimentos tienen la mayor fracción $(71,5 \%)$, seguidos de los residuos de jardinería (33,4\%), los residuos de madera (26,6 \%), el papel $(10,7 \%)$ y el cartón $(9,7 \%)$. Materia volátil: destacan los residuos de jardín con mayor proporción de volátiles $(89,0 \%)$, seguidos del cartón $(87,6 \%)$, residuos de alimentos (82,3\%), papel $(81,7 \%)$, y la madera $(80,00 \%)$. Contenido de cenizas: los residuos de alimentos destacan con mayor cantidad de cenizas $(15,8 \%)$, seguidos del papel $(7,8 \%)$, madera $(4,8 \%)$, y jardinería y cartón (3, $5 \%)$. Carbono fijo: se destaca la madera con mayor cantidad de carbón (15,2\%), seguida del papel 10,5\%), el cartón 8,9\%, jardinería (7,6\%) y desperdicio de alimentos (1,9\%).

\section{Tabla 3}

Análisis de la fracción degradable de residuos domésticos en Santiago de los Caballeros, 2019

\begin{tabular}{ccccc}
\hline Tipo de residuo & $\begin{array}{c}\text { Contenido de } \\
\text { humedad (\%) }\end{array}$ & $\begin{array}{c}\text { Contenido } \\
\text { volátil (\%) }\end{array}$ & $\begin{array}{c}\text { Contenido de } \\
\text { cenizas (\%) }\end{array}$ & $\begin{array}{c}\text { Carbono } \\
\text { fijo (\%) }\end{array}$ \\
\hline Residuos de alimentos & 71,46 & 82,25 & 15,83 & 1,92 \\
\hline Residuos de jardinería & 33,44 & 88,95 & 3,50 & 7,55 \\
\hline Madera & 26,58 & 80,00 & 4,78 & 15,22 \\
\hline Papel & 10,70 & 81,68 & 7,83 & 10,49 \\
\hline Cartón & 9,69 & 87,60 & 3,50 & 8,90 \\
\hline
\end{tabular}




\section{Poder calorífico}

La tabla 4 muestra el valor calorífico más alto correspondiente para cada tipo de residuo degradable con su desviación estándar. El HHV promedio es $9.337,6 \mathrm{~kJ} \cdot \mathrm{kg}^{-1}$, mostrando los residuos de jardinería el valor más alto con $10.560,6 \mathrm{~kJ} \cdot \mathrm{kg}^{-1}$, seguido de desperdicios de alimentos con 10.385, $4 \mathrm{~kJ} \cdot \mathrm{kg}^{-1}$, desperdicios de madera, cartón y papel con $9.875,0 \mathrm{~kJ} \cdot \mathrm{kg}^{-1}$ y $9.227,4 \mathrm{~kJ} \cdot \mathrm{kg}^{-1}$ y $6.639,7 \mathrm{~kJ} \cdot \mathrm{kg}^{-1}$, respectivamente.

\section{Tabla 4}

Poder Calorifico Superior de la fracción degradable de residuos domésticos en Santiago de los Caballeros 2019

\begin{tabular}{lrrr}
\hline Tipo de residuo & HHV $(\mathrm{kJ} / \mathrm{kg})$ & + D.S & \\
\hline Desperdicio de alimentos & $10.385,4$ & \pm & 285,1 \\
\hline Residuos de jardinería & $10.560,6$ & \pm & 243,3 \\
\hline Madera & $9.875,0$ & \pm & $1.062,1$ \\
\hline Cartón & $9.227,4$ & \pm & 296,5 \\
\hline Papel & $6.639,7$ & \pm & $1.378,6$ \\
\hline Promedio & $9.337,6$ & \pm & $1.594,7$ \\
\hline Desviación estándar & & & \\
\hline
\end{tabular}

\section{Determinación del potencial eléctrico}

Para la determinación del potencial eléctrico se utilizaron los valores obtenidos de los residuos promedio y se extrapolaron con la población número: 829,899 habitantes de Santiago, obtenida en el censo nacional (Oficina Nacional de Estadística, 2010). La tabla 5 muestra el potencial de generación de energía eléctrica bruta para los diferentes tipos de desechos degradables. Cada tecnología contempla una eficiencia diferente según el tipo de residuo que se analiza, pero en circunstancias reales existen diferentes factores o variables como la humedad o el contenido de cenizas que inciden, por lo que las eficiencias pueden variar. El mayor potencial para la generación de residuos degradables combinados es la gasificación 
y ciclo combinado (GCC), alcanzando un máximo de aproximadamente 308,8 MWh por día, seguido del proceso de pirólisis de ciclo combinado (PCC), con un potencial de 273, $5 \mathrm{MWh}$. En cuanto a la tecnología de gasificación acoplada a un ciclo de turbina de gas (GTG), tiene un potencial de generación aproximado de 220,6 MWh por día y de Combustión Directa (DC), un potencial de 175,6 MWh.

\section{Tabla 5}

Potencial bruto de generación eléctrica de la fracción degradable de residuos domésticos en Santiago de los Caballeros, 2019

\begin{tabular}{lrrrr}
\hline Tipo de residuo & $\begin{array}{l}\text { MWh } \\
\text { (DC) }\end{array}$ & $\begin{array}{l}\text { MWh } \\
(\text { GGT) }\end{array}$ & $\begin{array}{r}\text { MWh } \\
\text { (CCG) }\end{array}$ & $\begin{array}{r}\text { MWh } \\
(\text { PCC })\end{array}$ \\
\hline Desperdicio de alimentos & 102,24 & 128,44 & 179,82 & 159,27 \\
\hline Residuos de jardinería & 48,66 & 61,13 & 85,59 & 75,81 \\
\hline Madera & 6,70 & 8,41 & 11,78 & 10,43 \\
\hline Cartón & 11,54 & 14,50 & 20,30 & 17,98 \\
\hline Papel & 6,43 & 8,08 & 11,31 & 10,02 \\
\hline Total & 175,57 & 220,57 & 308,80 & 273,50 \\
\hline *DC= Combustión directa, GGT=Gasificación con turbina de gas, & \\
GCC= Gasificación con ciclo combinado, PCC= Pirolisis con ciclo combinado \\
\hline
\end{tabular}

\section{Discusión}

Los resultados presentados en la tabla 2 muestran que la fracción de residuos biodegradables y no biodegradables encontrada en esta investigación es muy similar a la realizada en 2006 por el Ayuntamiento de Santiago de los Caballeros, donde se obtuvo que la composición fue $65,7 \%$ biodegradable y $34,3 \%$ no biodegradable y peligroso. También se encontró evidencia de otra investigación realizada en San Francisco de Macorís en 2017, en la que la composición de los residuos sólidos varió levemente, encontrándose $71,8 \%$ para residuos orgánicos y $28,2 \%$ para residuos inorgánicos y peligrosos. Estas similitudes pueden deberse a la proximidad de ambas ciudades. En cuanto a la composición de cada grupo, también se observa una similitud de $62,3 \%$ para desperdicio de 
alimentos y 9,5 \% para desperdicio de jardín, 0,5 \% papel y 1,7 \% cartón (Ureña, 2017).

Los valores encontrados para el mayor poder calorífico que se muestran en la tabla 3 son superiores a algunos estudios realizados en la ciudad de Bogotá en 2011, donde los residuos tuvieron un poder calorífico promedio mayor de $1.153,5 \mathrm{~kJ} \cdot \mathrm{kg}^{-1}$. Esto puede estar asociado con el hecho de que la prueba de valor calorífico en este estudio se realizó en base seca y la suya en base húmeda (Alcaldía Mayor de Bogotá, 2011). Se sustenta que para una correcta combustión el valor calorífico inferior no debe ser menor a 7.000 $\mathrm{kJ} \cdot \mathrm{kg}^{-1}$ de media al año (Mutz, 2017). Este resultado parece ofrecer buenas indicaciones, ya que los valores obtenidos para este estudio se mantienen en ese orden, con $9.155,4 \mathrm{~kJ} \cdot \mathrm{kg}^{-1}$.

Los resultados obtenidos en este estudio, residuos alimento 10.385,4 $\mathrm{kJ} \cdot \mathrm{kg}^{-1}$, residuos de jardinería $10.560,6 \mathrm{~kJ} \cdot \mathrm{kg}^{-1}$, madera $9.875,0 \mathrm{~kJ} \cdot \mathrm{kg}^{-1}$, papel $9.227,4 \mathrm{~kJ} \cdot \mathrm{kg}^{-1}$ y cartón $6.639,7 \mathrm{~kJ} \cdot \mathrm{kg}^{-1}$ al compararse de forma individual, se observa que los valores varían con otras investigaciones que se han realizado, como en el estudio de González, desarrollado en el 2007, donde los residuos orgánicos que contienen un conjunto alimentos y residuos de jardinería rondan $4.184,0 \mathrm{~kJ} \cdot \mathrm{kg}^{-1}$, siendo más que el doble para este estudio realizado. Respecto a la madera, el valor obtenido es $14.225,68 \mathrm{~kJ} \cdot \mathrm{kg}^{-1}$ superando claramente casi en $50 \%$ el valor obtenido en este estudio (González, 2007). En otro estudio presentado por Kokalj y Samec en el 2019, obtuvieron resultados para los residuos de alimentos $10.100,0 \mathrm{~kJ} \cdot \mathrm{kg}^{-1}$, muy similar al resultado obtenido en esta investigación. En cuanto a la madera, con $16.320 \mathrm{~kJ} \cdot \mathrm{kg}^{-1}$, y cartón, con $17.490 \mathrm{~kJ} \cdot \mathrm{kg}^{-1}$, ambos presentan valores muy superiores a los obtenidos en este estudio (Kokalj \& Samec, 2019).

Estas diferencias en los residuos de alimentos podrían atribuirse a los hábitos de consumo asociados a la alimentación y a la variedad de productos que son desechados en los alimentos. En cuanto a los residuos de jardinería y madera, se podría inferir que las diferencias son por los tipos de especies en jardinería y procedencias de las maderas, así como al contenido de humedad. Sobre el cartón y papel, una posible causa de 
la variabilidad es la cantidad de tipos de papel y cartón disponibles en el mercado. Al analizar el potencial de los residuos, se evidencia que el poder calorífico es bajo, comparado con combustibles sólidos como el carbón vegetal con $31.980 \mathrm{~kJ} \cdot \mathrm{kg}^{-1}$ o el carbón mineral con $29.300 \mathrm{~kJ} \cdot \mathrm{kg}^{-1}$ (Fuwape \& Akindele, 1997; KEMA, 1997). Es por esto por lo que las tecnologías más eficientes tienen el mayor potencial de generación para compensar el menor poder calorífico asociado a los residuos.

La tabla 5 muestra el potencial de generación de energía eléctrica bruta para cada tipo de residuo, dependiendo de la tecnología de conversión de energía. Asociado a estos valores, si es posible garantizar la homogeneidad en los residuales, los valores estarán aún más cerca de los calculados. La humedad de los residuos podría ser un problema para cualquiera de las tecnologías consideradas en esta investigación, ya que un alto contenido de agua afecta la ignición del combustible y reduce el poder calorífico de los productos gaseosos, debido al gasto energético requerido para la evaporación del agua antes de realizar la combustión o gasificación de la biomasa, reduciendo consecuentemente el rendimiento de calor o energía del combustible (López-Chalarca et al., 2020). Sería muy ventajoso analizar un método de secado natural en el que se pueda reducir la humedad sin consumir energía del propio proceso. Considerando las condiciones climáticas en República Dominicana, se podría implementar un método de secado natural (Organización de Productividad de Asia, 2009; Serrano, 2017).

Una investigación realizada en Ecuador calculó que el porcentaje de energía de evaporación es casi equivalente al porcentaje de humedad. Destaca su ejemplo con el cacao, donde el $83 \%$ de la energía generada se consume en el proceso de secado. Esta información podría estar relacionada con el alto contenido de humedad en los residuos de alimentos para Santiago de los Caballeros, con 71,5 \% (Serrano, 2017).

La materia volátil es la porción de gases condensables y no condensables que se liberan cuando un compuesto se somete a una temperatura específica en un tiempo determinado (Hidalgo et al, 2015). Comparando los resultados de este estudio, residuos de alimentos $82,25 \%$, residuos de 
jardinería 88,95\%, madera $80.0 \%$, cartón 81,68 \% y papel 87,60 \%, con un estudio realizado en 2015, donde se evaluaron los mismos parámetros, se observan valores, en todos los casos, ligeramente inferiores: residuos de alimentos con $80 \%$, residuos de jardinería 75,0 \%, cartón 76,0 \% y el papel 84,0 \% (Kalanatarifard \& Yang, 2012). Para otro estudio realizado en 2014, al comparar con los resultados de este estudio, solo los residuos de alimentos con 87,3\% son ligeramente superiores. Por el contrario, son el papel, 78,9\%, y el cartón, 79,8 \%, ligeramente inferiores (Zhou, 2014). López-Chalarca et al. (2020) reportaron valores de $71.8 \%$ de materia volátil (base húmeda) en madera de Acacia mangium, siendo inferiores a los encontrados en este estudio. Según Telmo et al. (2010), el contenido de materia volátil en la biomasa ronda entre 76 y $86 \%$ en base seca.

La ceniza es el residuo sólido inorgánico que resulta después de quemar completamente el combustible, contiene metales alcalinos, sílice y otros materiales inorgánicos (Hidalgo et al., 2015). Comparando el contenido de cenizas obtenidos en este estudio residuos de alimentos 15,8 \%, residuos de jardinería 3,5 \%, cartón 3,5 \% y papel 7,8 \% con el estudio de Kalanatarifard y Yang en 2012, solo los residuos alimento, $14,0 \%$, presentan un valor ligeramente inferior en comparación a este estudio. El cartón, 12,0 \%, y papel, $9 \%$, son considerablemente superiores. Para el estudio hecho por Zhou en 2014, los residuos de alimentos, 0,6\%, son, con una gran diferencia, inferiores a los obtenidos en este estudio. Los residuos de jardinería $20 \%$, papel $9 \%$ y cartón $7,22 \%$ son en contraste superiores, respectivamente (Kalanatarifard \& Yang, 2012; Zhou, 2014).

Para el cálculo del carbono fijo en la madera se obtuvieron resultados comparables para maderas específicas, que oscilan entre $12,4 \%$ a 23,2 \% para las especies Acacia pennatula (Hidalgo et al., 2015) y A. mangium (Marsoem \& Irawati, 2016), respectivamente. Muy cercano a los valores obtenidos para este estudio, con 15,2\%. Los bosques encontrados correspondieron a especies como Leucaena leucocephala y Delonix regia, además de otras especies decorativas. Comparado con un estudio municipal realizado por Zhou, en 2014, con diferentes residuos municipales, el papel obtuvo un 9,7\%, ligeramente inferior al realizado en este estudio con 
10,5 \%, Cartón 13,3 \% superior en comparación 8,9 \%, jardinería 17,2 \% mayor con 7,6 \% y residuos de alimentos con un 10,3\% en contraste con $1,9 \%$, siendo todos muy superiores a los realizados en este estudio (Zhou, 2014).

Un mayor contenido de carbono fijo y volátil representa una mayor cantidad de energía en la materia. Un aspecto importante para considerar es la sostenibilidad de la producción de energía (Hidalgo et al., 2015). El alto contenido de cenizas disminuye el poder calorífico de las sustancias y requiere una extracción continua, lo que se traduce en un incremento de los precios. Los componentes más presentes en las cenizas son $\mathrm{Si}, \mathrm{Ca}$, $\mathrm{Mg}, \mathrm{K}, \mathrm{Na}, \mathrm{Cl}, \mathrm{P}$, y por efecto de la temperatura tienden a reaccionar provocando problemas en los sistemas de intercambio de calor. Algunos metales pesados se pueden encontrar en concentraciones mucho más bajas (Fe, Al, Mn y Ti) (Hidalgo et al., 2015).

\section{Conclusiones}

De acuerdo con los resultados de esta investigación se obtuvieron las siguientes conclusiones: el valor calorífico de la fracción degradable de los residuos sólidos producidos en la ciudad de Santiago de los Caballeros estuvo en un rango superior al esperado para estos residuos, siendo de mayor a menor en el siguiente orden: residuos de jardinería, residuos de alimentarios, residuos de madera, cartón y papel. El potencial bruto de generación eléctrica calculado alcanzó un máximo de aproximadamente 308,8 MWh por día, utilizando el método de Gasificación con Ciclo Combinado (GCC), Pirólisis y Ciclo Combinado (PCC) 273,50 MWh. El desperdicio de alimentos se suma a la mayor parte del potencial energético y a menos que se considere un método de secado eficiente y un equipo de alta tolerancia a las cenizas, se consideran los residuos de alimentos no adecuados para estas tecnologías. El contenido de humedad de los residuos de alimentos fue del 71,46\% y los residuos de jardinería del 33,44\%. La mayoría de las tecnologías toleran un máximo del $30 \%$.

Se recomienda buscar un método de secado natural donde se pueda reducir la humedad sin consumir energía del proceso y aprovechando 
las condiciones climáticas del país. El alto contenido de cenizas en los residuos alimentarios con un 15,83 \%, hace que su manipulación sea todo un reto. Con base en los resultados se podría concluir que los residuos alimentarios no son adecuados para tecnologías de conversión termoquímica. Se recomienda realizar un estudio sobre el potencial de generación de energía con tecnologías de transformación bioquímica como el biogás. Es de esperar que comprender el potencial de la generación de energía a partir de residuos, especialmente de la fracción degradable, arroje luz sobre estrategias para mejorar la gestión de residuos y la matriz energética de República Dominicana.

\section{Reconocimientos}

Los autores agradecen a la Universidad ISA (UNISA), Universidad Abierta para Adultos (UAPA), Universidad Católica Nordestana (UCNE) y FONDOCYT. Así como también a las personas envueltas en proceso, directivas, vicerrectorías que apoyaron la gestión del proyecto en especial al Dr. Nelson Abreu, investigador principal del proyecto. Este trabajo fue apoyado por la subvención del FONDOCYT, República Dominicana (No 2016-2017-092) y el Consorcio de Universidades mencionado anteriormente. La prueba calorimétrica se realizó en el Laboratorio de Energía Renovable de la Universidad ISA.

\section{Referencias}

Alcaldía Mayor de Bogota. (2011). Caracterización de los residuos sólidos residenciales generados en la ciudad de Bogota D.C 2011. Bogotá.

Ayuntamiento del Municipio de Santiago de los Caballeros. (2006). Análisis de Composición de Residuos.

Banco Mundial. (2019). Consumo de energía procedente de combustibles fósiles (\% del total). Obtenido de Consumo de energía procedente de combustibles fósiles (\% del total): https://datos.bancomundial. org/indicador/EG.USE.COMM.FO.ZS?view=chart

Breton, D., Rodríguez, L., Pérez, R., y Arteaga, L. (2012). Métodos de Estimación de Biomasa Potencial. https://www.researchgate. net/publication/280086387_Metodos_de_estimacion_de_la_ biomasa_potencial 
Comisión Nacional de Energía -CNE. (2009). Comisión Nacional de Energía. http://www.cne.gov.do/, 19/08/2018.

Comisión Nacional de Energía -CNE. (2017). Contexto energético de la República Dominicana. https:/www.cne.gob.do/wp-content/ uploads/2017/07/Boletin-Contexto-Energetico.pdf

Dieter Mutz, D. H. (2017). Waste-to-Energy Options in Municipal Solid Waste Management. Eschborn: Deutsche Gesellschaft für Internationale Zussamenarbeit (GIZ).

Dutta, P., Das, A., Pandey, V., Devi, M. (2014). Características de combustible de algunas biomasas disponibles localmente como una materia prima de gasificación potencial para la aplicación térmica. Investigación quimica industrial e ingeniería, 5, 19806-19813

Fernández, J. (9 de septiembre de 2019). Poder Calorífico, Máquinas Térmicas. http://www.edutecne.utn.edu.ar/maquinas_termicas/ 01-poder_calorifico.pdf

Fuwape, J. A. \& Akindele, S. O. (1997). Rendimiento de biomasa y valor energético de algunos árboles multiusos de rápido crecimiento en Nigeria. Biomasa y Bioenergía, 12, 101-106.

Gautam, G., S. A. (2014). Gasificación de astillas de madera, residuos agrícolas y residuos en un gasificador de corriente descendente comercial. Sociedad Americana de Ingenieros Agrícolas y Biológicos, 2-4.

González J. (2007). Módulo I: Contaminación Ambiental, Residuos Sólidos Urbanos. EOI Escuela de Negocios.

Hidalgo, F. A., Honorato, J. A., \& Hernández, G. C. (2015). Caracterización Energética de la madera de Acacia pennatula Schltdl. \& Cham y Trema micrantha(L.) Blume. Revista Mexicana de Ciencias Forestales, 71-81.

Kalanatarifard, A., \& Yang, G. S. (2012). Identificación de las características municipales de los residuos sólidos y el potencial de recuperación plástica en el vertedero de Bakri, Muar, Malasia. Revista de Desarrollo Sostenible, 15-16.

KEMA. (1997). Informe de impacto ambiental, Inflamación de lodos de aguas residuales secas en el Hemwegcentrale de Amsterdam. KEMA 64652-KES/MAD 96-3037.

Kokalj, F., Samec, N. (2013). Combustion of Municipal Solid Waste for Power Production, (pp. 280). http://dx.doi.org/10.5772/55497. 
López-Chalarca, L. T., Vega Rodríguez, L. Y., Rendón Colorado, C. D., y Tobón-Rojas, S. (2020). Caracterización de los residuos de la industria maderera para su aprovechamiento en diferentes aplicaciones. Revista Científica Ingeniería y Desarrollo, 38(1), 104-124.

Marsoem, N., y Irawati, D. (2016). Basic properties of Acacia mangium and Acacia auriculiformis as a heating fuel. Advances of Science and Technology for Society. https://aip.scitation.org/doi/abs/10.10 $63 / 1.4958551$

McClave, J., Dietrich, F. (1979). Distribuciones de muestreo. En Estadistica (pp. 201-217). San Francisco.

Ministerio de Medio Ambiente y Recursos Naturales. (2014). Proyecto de Fortalecimiento de la Capacidad Institucional en el Manejo Integral de los Residuos Sólidos a Nivel Nacional en la República Dominicana.

Oficina Nacional de Estadística (2010). IX Censo de Población y Vivienda 2010 - República Dominicana. https://www.one.gob.do/censos/ poblacion-y-vivienda/censo-2010.

Organización Asiática de Productividad. (2009). Biomasa como combustible en calderas pequeñas.

Organización de las Naciones Unidas para la Alimentación y la Agricultura. (2018). Análisis Especial de Balance. Colección de documentos técnicos.

Serrano, J. (2017). Determinación del Potencial de Generación Eléctrica a partir de Biomasa en el Ecuador. Revista de la Facultad de Ciencias Quimicas, 52-55.

Steffen, R., y Szolar, O. A. (1998). Materias primas para digestión anaeróbica. Instituto de Agrobiotecnología Tulln Universidad de Ciencias Agrícolas de Viena.

Telmo, C., Lousada, J., \& Moreira, N. (2010). Proximate analysis, backwards stepwise regression between gross calorific value, ultimate and chemical analysis of wood. Bioresource technology, 101(11), 3808-3815. https://www.sciencedirect.com/science/article/abs/pii/ S0960852410000787

The Economist Intelligence Unit. (2015). The future of the electric sector in Dominican Republic. https:/www.ces.org.do/images/2015/ FunglodeElectricitySectorReportEnglishFINAL.pdf 
United Nations Climate Change. (2015). United Nations Climate Change. https://unfccc.int/process-and-meetings/the-paris-agreement/ the-paris-agreement

Ureña, F. (2017). Diagnóstico de la Situación de la Gestión de Residuos Sólidos del Municipio de San Francisco de Macorís. [Tesis para obtener título de Magister en Ingeniería Sanitaria y Ambiental, Instituto Tecnológico de Santo Domingo].

Worldwatch Institute. (2015). Aprovechar los recursos energéticos sostenibles de la República Dominicana. Washington.

Zhou, H. (2014). Clasificación y comparación de residuos sólidos municipales en función de las características termoquímicas. Diario de la Asociación de Gestión de Residuos y Aire, 597-612. 
